\title{
Prevalence and progression of subclinical atherosclerosis in patients with chronic kidney disease and diabetes
}

\section{Palanca, Ana}

2018-09

Palanca , A, Castelblanco , E , Perpinan , H, Betriu , A, Soldevila, B , Manuel Valdivielso , $\mathrm{J}$, Bermudez, M , Duran , X , Fernandez , E , Puig-Domingo , M , Groop , P-H , Alonso , N \& Mauricio , D 2018 , ' Prevalence and progression of subclinical atherosclerosis in patients with chronic kidney disease and diabetes ' , Atherosclerosis , vol. 276 , pp. 50-57 . https://doi.org/10.1016/j.atheroscl

http://hdl.handle.net/10138/305122

https://doi.org/10.1016/j.atherosclerosis.2018.07.018

publishedVersion

Downloaded from Helda, University of Helsinki institutional repository.

This is an electronic reprint of the original article.

This reprint may differ from the original in pagination and typographic detail.

Please cite the original version. 


\title{
Prevalence and progression of subclinical atherosclerosis in patients with chronic kidney disease and diabetes
}

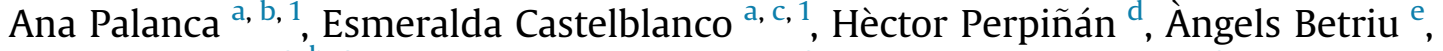 \\ Berta Soldevila a, b, c, José Manuel Valdivielso e , Marcelino Bermúdez ${ }^{\mathrm{e}}$, Xavier Duran ${ }^{\mathrm{f}}$, \\ Elvira Fernández e, Manel Puig-Domingo a, b, c, Per-Henrik Groop ${ }^{\text {g, h, i, j, }}$

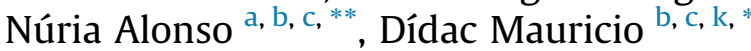 \\ a Department of Endocrinology and Nutrition, Health Sciences Research Institute \& University Hospital Germans Trias i Pujol, Badalona, Spain \\ ${ }^{\mathrm{b}}$ Department of Medicine, Barcelona Autonomous University (UAB), Barcelona, Spain \\ ${ }^{\mathrm{c}}$ Center for Biomedical Research on Diabetes and Associated Metabolic Diseases (CIBERDEM), Spain \\ d Biostatistics Unit, Institut de Recerca Biomèdica de Lleida, Spain \\ e Vascular and Renal Translational Research Group. Institut de Recerca Biomèdica de Lleida, Spain \\ ${ }^{\mathrm{f}}$ Methodological and Biostatistical Advisory Service, Institut Hospital del Mar d'Investigacions Mèdiques, Barcelona, Spain \\ ${ }^{g}$ Folkhälsan Institute of Genetics, Folkhälsan Research Center, Helsinki, Finland \\ h Abdominal Center Nephrology, University of Helsinki and Helsinki University Central Hospital, Helsinki, Finland \\ ${ }^{\mathrm{i}}$ Research Programs Unit, Diabetes and Obesity, University of Helsinki, Helsinki, Finland \\ ${ }^{\mathrm{j}}$ Baker IDI Heart and Diabetes Institute, Melbourne, Australia \\ ${ }^{\mathrm{k}}$ Department of Endocrinology and Nutrition, University Hospital de la Santa Creu i Sant Pau \& Institut d'Investigació Biomédica Sant Pau (IIB Sant Pau), \\ Barcelona, Spain
}

\section{A R T I C L E I N F O}

\section{Article history:}

Received 5 April 2018

Received in revised form

21 June 2018

Accepted 12 July 2018

Available online 19 July 2018

\section{Keywords}

Chronic kidney disease

Diabetes

Subclinical atherosclerosis

Cardiovascular outcomes

\begin{abstract}
A B S T R A C T
Background and aims: Cardiovascular disease is the leading cause of morbidity and mortality in patients with chronic kidney disease (CKD) and diabetes. Traditional cardiovascular risk factors fail to fully account for the increase in cardiovascular risk in these patients. This study aims to analyse the prevalence and progression of subclinical atherosclerosis in CKD patients with and without diabetes.

Methods: We included data from CKD patients with and without diabetes free from previous cardiovascular events from the NEFRONA cohort. Patients underwent baseline and 24-month follow-up carotid and femoral ultrasound examinations. Multivariable models were used to assess the contribution of diabetes to the presence and plaque progression.

Results: A total of 419 patients with diabetes and 1129 without diabetes were included. Diabetic patients were older, had higher BMIs, more hypertension and dyslipidaemia. At baseline, the proportion of patients with plaque was higher among diabetic patients $(81.4 \% v s .64 .1 \%, p<0.001)$. Diabetic patients more frequently had more than two vascular territories with plaque $(64.4 \% v s .48 .4 \%, p<0.001)$. Multivariable analysis indicated that plaque at baseline was significantly associated with age, gender, smoking and renal replacement therapy (RRT) in the non-diabetic patients, but only with age and male gender in diabetic patients. Plaque progression was significantly associated with age, number of territories with basal plaque, smoking and RRT in both groups.

Conclusions: Subclinical atherosclerosis is more prevalent, carries a higher plaque burden and is more rapidly progressive in renal patients with diabetes. In these patients, diabetes outweighs other described risk factors associated with the presence of subclinical atherosclerosis.
\end{abstract}

(c) 2018 Elsevier B.V. All rights reserved.

\footnotetext{
* Corresponding author. Department of Endocrinology and Nutrition, Hospital de la Santa Creu i Sant Pau, Carrer de Sant Quintí, 89, 08041, Barcelona, Spain.

** Corresponding author. Department of Endocrinology and Nutrition, University Hospital Germans Trias i Pujol, Carretera Canyet S/N, 08916, Badalona, Spain. E-mail addresses: nalonso32416@yahoo.es (N. Alonso), didacmauricio@gmail.com (D. Mauricio).

1 These authors contributed equally to this work.
} 


\section{Introduction}

Cardiovascular disease (CVD) is the leading cause of morbidity and mortality in patients with chronic kidney disease (CKD) and in patients with diabetes mellitus (DM) [1,2]. Both conditions are considered to confer high or very high CVD risk in major guidelines [3]. Commonly used CVD risk scores and prediction models perform poorly in CKD [4] and in patients with diabetes [5]. Additionally, in patients with type 2 diabetes, impaired renal function and albuminuria are independently associated with the incidence of CV events [6-10]. Several studies have reported an increase in the incidence and severity of coronary heart disease as glomerular filtration rate (GFR) declines [11-13]. CKD is estimated to affect $50 \%$ of patients with diabetes globally, and its presence and severity influences disease prognosis [14]. A large prospective study of over 4000 individuals with type 1 diabetes in Finland found independent associations between the presence and severity of kidney disease and cardiovascular mortality [15].

The natural history of atheromatous disease involves a prolonged silent phase, and disease is often not detected until advanced stages, usually following a CV event. Patients with diabetes present a higher prevalence of carotid plaques and more advanced preclinical carotid atherosclerosis than non-diabetic subjects, even after controlling for traditional CV risk factors [16]. The detection of subclinical atherosclerosis through non-invasive methods, such as arterial ultrasonography, has been demonstrated to be useful for the prediction of future CV events [17-19].

Recent data showed that the presence of carotid plaques and their volume or total area shows higher accuracy for predicting CV events than carotid intima media thickness (cIMT) [20,21]. Furthermore, different studies have demonstrated the value of evaluating subclinical atherosclerosis in multiple territories for $\mathrm{CV}$ event prediction [22-25].

The National Observatory of Atherosclerosis in Nephrology (NEFRONA) study is a large, multicentre, prospective, observational study that evaluated atheromatous disease in the carotid and femoral territories in a large cohort of Spanish patients with CKD without previous CVD [26,27]. Recently, the NEFRONA study demonstrated that patients in the early and late stages of kidney disease show a higher prevalence of plaques [28-30]. Fewer data exist on the prevalence of subclinical atherosclerosis in patients with CKD and diabetes. We hypothesized that DM involves an additional CVD burden in patients with CKD. Therefore, the aim of our study was to analyse the prevalence and progression of subclinical atheromatous disease in CKD patients with and without DM in this cohort.

Table 1

Clinical and anthropometrical characteristics of the baseline population.

\begin{tabular}{|c|c|c|c|}
\hline & CKD without diabetes & CKD with diabetes & $p$-value \\
\hline & $\mathrm{N}=1129$ & $\mathrm{~N}=419$ & \\
\hline Gender (male) & $677(60.0 \%)$ & $277(66.1 \%)$ & 0.032 \\
\hline Age (years) & $60.0[49.0 ; 67.0]$ & $65.0[57.5 ; 70.0]$ & $<0.001$ \\
\hline Race (white/other) & $1095(97.0 \%)$ & $409(97.6 \%)$ & 0.628 \\
\hline Smoking habit & $620(54.9 \%)$ & $244(58.2 \%)$ & 0.267 \\
\hline Hypertension & $1051(93.1 \%)$ & $410(97.9 \%)$ & $<0.001$ \\
\hline Dyslipidaemia & $733(64.9 \%)$ & $329(78.5 \%)$ & $<0.001$ \\
\hline CKD stages: & & & 0.002 \\
\hline Stage 3 & $501(44.4 \%)$ & $204(48.7 \%)$ & \\
\hline Stage $4-5$ & $411(36.4 \%)$ & $166(39.6 \%)$ & \\
\hline RRT & $217(19.2 \%)$ & $49(11.7 \%)$ & \\
\hline CKD etiology: & & & $<0.001$ \\
\hline Diabetic nephropathy & $0(0 \%)$ & 199 (47.5\%) & \\
\hline Vascular nephropathy & $260(23.0 \%)$ & $74(17.7 \%)$ & \\
\hline Other & $869(77.0 \%)$ & $146(34.8 \%)$ & \\
\hline Glomerular filtration rate $(\mathrm{ml} / \mathrm{min})$ & $31.8[21.4 ; 44.1]$ & $33.4[22.4 ; 45.8]$ & 0.228 \\
\hline Creatinine $(\mathrm{mg} / \mathrm{dL})$ & $2.04[1.54 ; 2.90]$ & $1.99[1.55 ; 2.76]$ & 0.28 \\
\hline Urea $(\mathrm{mg} / \mathrm{g})$ & $89.7[62.0 ; 124]$ & $88.0[60.0 ; 120]$ & 0.251 \\
\hline Body Mass Index $\left(\mathrm{kg} / \mathrm{m}^{2}\right)$ & $27.6[24.7 ; 31.0]$ & $30.0[26.6 ; 33.7]$ & $<0.001$ \\
\hline Waist/hip ratio $(\mathrm{cm})$ & $96.0[89.0 ; 105]$ & $103[94.0 ; 111]$ & $<0.001$ \\
\hline Uric acid (mg/dL) & $6.67[5.60 ; 7.80]$ & $6.70[5.80 ; 7.80]$ & 0.306 \\
\hline $\operatorname{ALT}(\mathrm{U} / \mathrm{L})$ & $18.0[14.0 ; 25.1]$ & $19.9[15.0 ; 27.2]$ & 0.001 \\
\hline AST (U/L) & $19.9[16.0 ; 24.0]$ & $20.0[17.0 ; 26.0]$ & 0.052 \\
\hline hs C-reactive protein (mg/L) & $1.79[0.90 ; 3.92]$ & $2.30[1.00 ; 5.40]$ & $<0.001$ \\
\hline Systolic blood pressure (mmHg) & $139[127 ; 154]$ & $146[131 ; 162]$ & $<0.001$ \\
\hline Diastolic blood pressure (mmHg) & $82.0[75.0 ; 89.0]$ & $79.0[71.0 ; 87.0]$ & $<0.001$ \\
\hline Pulse pressure (mmHg) & $56.0[47.0 ; 68.0]$ & $68.0[55.0 ; 80.0]$ & $<0.001$ \\
\hline Total cholesterol (mg/dL) & $180[156 ; 207]$ & $173[146 ; 198]$ & $<0.001$ \\
\hline HDL cholesterol (mg/dL) & $48.0[40.0 ; 59.0]$ & $43.6[36.0 ; 53.0]$ & $<0.001$ \\
\hline LDL cholesterol (mg/dL) & $105[84.0 ; 125]$ & $93.0[73.8 ; 114]$ & $<0.001$ \\
\hline Non-HDL cholesterol (mg/dL) & $129[108 ; 154]$ & $122[102 ; 148]$ & 0.007 \\
\hline Triglycerides (mg/dL) & $118[89.0 ; 162]$ & $141[102 ; 205]$ & $<0.001$ \\
\hline Glucose $(\mathrm{mg} / \mathrm{dL})$ & $94.0[86.0 ; 102]$ & $133[111 ; 162]$ & $<0.001$ \\
\hline HbA1c $(\%)$ & $5.50[5.10 ; 5.80]$ & $6.70[6.15 ; 7.75]$ & $<0.001$ \\
\hline Albumin/creatinine ratio $(\mathrm{mg} / \mathrm{g})$ & $83.5[12.0 ; 377]$ & $138[15.5 ; 471]$ & 0.036 \\
\hline Corrected calcium $(\mathrm{mg} / \mathrm{dL})$ & $9.20[8.86 ; 9.52]$ & $9.31[8.98 ; 9.68]$ & $<0.001$ \\
\hline Phosphate $(\mathrm{mg} / \mathrm{dL})$ & $3.70[3.27 ; 4.40]$ & $3.80[3.30 ; 4.30]$ & 0.695 \\
\hline 25 -Hydroxy vitamin $\mathrm{D}(\mathrm{pg} / \mathrm{mL})$ & $15.6[11.9 ; 20.1]$ & $14.1[10.5 ; 18.9]$ & $<0.001$ \\
\hline Antihypertensive treatment & $1006(89.1 \%)$ & $399(95.2 \%)$ & $<0.001$ \\
\hline Statin treatment & $660(58.5 \%)$ & $269(64.2 \%)$ & 0.047 \\
\hline Phosphate binder treatment & $339(30.0 \%)$ & $95(22.7 \%)$ & 0.005 \\
\hline Antiplatelet drug treatment & $201(17.8 \%)$ & $209(49.9 \%)$ & $<0.001$ \\
\hline
\end{tabular}

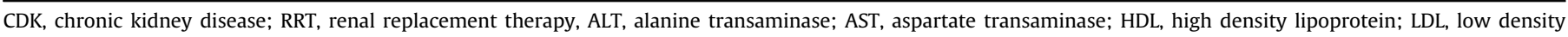
lipoprotein. 


\section{Materials and methods}

\subsection{Design and study population}

The design, methods, and baseline characteristics of the NEFRONA study have been published in detail elsewhere [26,27]. In brief, the NEFRONA study is a multicentre, prospective observational study designed to evaluate the subclinical atherosclerotic burden and the predictive value of carotid/femoral plaques in patients with CKD in Spain (stages 3-5). Exclusion criteria for both groups included: active infections, pregnancy, life expectancy lower than 12 months, history of clinical cardiovascular events, carotid artery surgery or any organ transplantation [28].

A total of 2445 CKD patients, 937 at CKD stage 3, 820 at CKD stage 4-5 and 688 on renal replacement therapy (RRT), all of them without previous cardiovascular disease at baseline were recruited from 81 Spanish hospitals between October 2010 and June 2012. A scheduled follow-up visit was arranged for 24 months after the baseline appointment. The present study was restricted to patients who attended both baseline and 24-month follow-up visits. Of the original NEFRONA study cohort, 893 subjects were excluded from the 24-month analysis due to death (46), cardiovascular event (80), receipt of renal allograft within the follow-up period (364), or second visit non-attendance (403). Another four patients were excluded because they had atheromatous plaques in all ten arterial territories at baseline. Overall, 1548 patients were included in the plaque progression analysis at 24 months. The study protocol was approved by the Ethics Committee of each hospital, and patients were included after signing informed consent.

\subsection{Clinical data and laboratory examinations}

Information regarding medical history, cardiovascular risk factors, hypertension, dyslipidaemia, diabetes and medication was collected at baseline. Dyslipidaemia was defined as a recorded clinical diagnosis or current use of lipid-lowering drugs. The physical examination included anthropometric measures (height, weight, waist-hip ratio (WHR)) and standard vital tests as previously described [26]. Biochemical data were obtained from a routine fasting blood test. The glomerular filtration rate (GFR) was estimated using the Modification of Diet in Renal Disease Study (MDRD-4) formula.

\subsection{Diagnosis of diabetes mellitus (DM)}

A patient was included in the DM group when one of the following criteria was met: (1) a diagnosis of DM was previously established and recorded in the patient's records, (2) fasting plasma glucose $\geq 126 \mathrm{mg} / \mathrm{dl}$ or $\mathrm{HbA} 1 \mathrm{c} \geq 6.5 \%$ identified by laboratory testing, or (3) the patient had a current prescription of any antidiabetic medication.

\subsection{Evaluation of $A D$ : ultrasound imaging}

B-mode ultrasound of carotid and femoral vascular sites was performed using the Vivid BT09 apparatus (General Electric) equipped with a 6-13 $\mathrm{MHz}$ broadband linear array probe as previously described [26]. The analysis of the presence of plaques was performed in ten vascular territories: internal, bulb and common carotid, and common and superficial femoral arteries by a single reader in a blinded fashion using the semi-automatic EchoPAC Dimension software (General Electric Healthcare). The presence of plaques was identified using B-mode and colour Doppler examinations and was defined as intima media thickness (cIMT) $>1.5 \mathrm{~mm}$ protruding into the lumen, according to the ASE Consensus Statement [31] and the Mannheim CIMT Consensus [32].

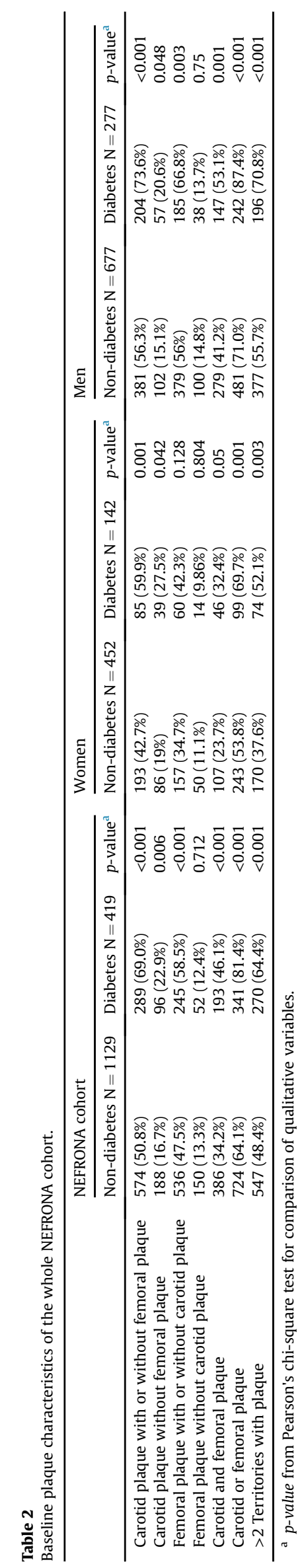




\subsection{Evaluation of progression of atheromatous disease}

Progression of atheromatous disease over 24 months was defined as an increase in the number of territories with plaque(s) compared to the baseline examination, as previously used in the MESA study [33].

\subsection{Statistical analysis}

Data are expressed as the means and standard deviations, medians and interquartile ranges, and absolute and relative frequencies as required. Patient characteristics were compared between nondiabetic patients and diabetic patients using Pearson's chi-square or Mann-Whitney U tests. The relationships between the presence of basal plaques and atherosclerosis progression at 24 months with potential risk factors were analysed using the Pearson chi-square test for qualitative variables and the Mann-Whitney $U$ test for quantitative variables. To assess the contribution of diabetes to the risk of having plaques and atherosclerosis progression, we fitted multivariable logistic regression models. Additional independent variables were included following a forward step procedure by means of likelihood ratio tests. Variables showing no statistical significance but changing any significant parameter by $>10 \%$ were considered confounders and were included in the model. To design the models of atheromatous disease (AD) progression, the variables entered were: gender, age in years (quadratic), race, smoking, dyslipidaemia, CKD stage, diabetes (only for whole population), body mass index (BMI), diastolic BP, pulse pressure, triglycerides, potassium, 25hydroxy vitamin D, C-reactive protein and number of territories with plaque(s) at baseline. Only significant variables in multivariate analysis in each group were included in the final model. To design the models of plaque presence, the variables entered were: gender, age in years (quadratic), race, smoking, hypertension, dyslipidaemia, CKD stage, diabetes (only for whole population), haemoglobin, uric acid, body BMI, diastolic BP, pulse pressure, HDL-cholesterol, LDLcholesterol, triglycerides, 25-hydroxy vitamin D, C-reactive protein, ALT, AST and ankle-brachial index. All tests were two-tailed at a statistical significance level of 0.05 . The statistical analysis was carried out with $\mathrm{R}$.

\section{Results}

\subsection{Baseline patient characteristics}

We included a total of 1548 patients, 419 with and 1129 without diabetes, for whom baseline and 24-month follow-up analysis were available. The baseline characteristics of the participants are shown in Table 1. Compared to non-diabetic patients, patients with diabetes were older and had higher BMI, WHR, triglycerides and serum hsCRP concentrations. However, diabetic patients had lower HDL and LDL cholesterol concentrations. A higher proportion of patients with hypertension or dyslipidaemia were observed in diabetic patients compared with non-diabetic patients. Up to $97.9 \%$ of patients with DM had hypertension and were on antihypertensive medication (95.2\%) and/or antiplatelet agents (49.9\%). Nondiabetic subjects were more frequently taking phosphate binders. Almost $19.2 \%$ of the non-diabetic patients were on renal replacement therapy (RRT) versus $11.7 \%$ of those with DM. Among the patients with diabetes, the foremost attributable causes of renal impairment were diabetic nephropathy (in 199 subjects, $47.5 \%$ ) and vascular nephropathy (in 74 subjects, $17.7 \%$ ).

\subsection{Baseline carotid and femoral atherosclerosis}

At baseline, the frequency of carotid plaques in any carotid territory (with or without the presence of femoral plaques) was significantly higher among patients with DM compared with patients without DM (69\% vs. 50.8\%, $p<0.001)$ (Table 2). The number of subjects with carotid plaques but without femoral plaques was also higher in the DM group $(22.9 \% v s .16 .7 \%, p=0.006)$. Furthermore, the frequency of femoral plaques at any site (with or without the presence of carotid plaques) was significantly higher in DM patients $(58.5 \%$ vs. $47.5 \%, p<0.001)$. However, no significant differences were found in the percentage of patients with femoral plaques but without carotid plaques. The proportion of patients with atherosclerotic plaques in both sites, carotid and femoral, was also higher in DM patients ( $46.1 \%$ vs. $34.2 \%, p<0.001)$. The prevalence of patients presenting plaques at either the carotid or the femoral site was significantly higher among patients with DM $(81.4 \%$ vs. $64.1 \%, p<0.001)$. The percentage of diabetic subjects with more than two vascular territories with plaque was also greater (64.4\% vs. 48.4\%, $p<0.001$ ).

Among men, carotid and femoral plaques were more common in those with DM than in those without DM (73.6\% vs. 56.3\%, $p<0.001 ; 66.8 \%$ vs. $56 \%, p=0.003$, respectively). Men with DM were more likely to present plaque at any vascular site $(87.4 \%$ vs. $71 \%, p<0.001)$. Similarly, plaques in more than two arterial territories were more common among men with DM (70.8\% vs. 55.7\%, $p<0.001$ ) (Table 2).

Among women, the prevalence of carotid plaques was higher in

Table 3

Multivariate logistic regression model for the presence of plaque in the whole NEFRONA cohort, CKD without diabetes and CKD without diabetes.

\begin{tabular}{|c|c|c|c|c|c|c|c|c|c|}
\hline & \multicolumn{3}{|c|}{ Whole NEFRONA cohort } & \multicolumn{3}{|c|}{ CKD without diabetes } & \multicolumn{3}{|c|}{ CKD with diabetes } \\
\hline & Estimate (SE) & OR $(95 \% \mathrm{CI})^{\mathrm{a}}$ & $p$-value & Estimate (SE) & OR $(95 \% \mathrm{CI})^{\mathrm{a}}$ & $p$-value & Estimate (SE) & OR $(95 \% \mathrm{CI})^{\mathrm{a}}$ & $p$-value \\
\hline Intercept & $-7.608(1.663)$ & na & 0.001 & $-6.910(1.812)$ & na & 0.001 & $-7.278(3.459)$ & na & 0.035 \\
\hline Gender (male) & $-0.909(0.737)$ & -- & 0.218 & $-1.982(0.830)$ & -- & 0.017 & $1.054(0.324)$ & $2.87(1.53,5.47)$ & 0.001 \\
\hline Age & $0.246(0.057)$ & -- & $<0.001$ & $0.230(0.064)$ & -- & 0.001 & $0.248(0.122)$ & -- & 0.042 \\
\hline $\mathrm{Age}^{2}$ & $-0.002(0.000)$ & -- & 0.004 & $-0.001(0.001)$ & -- & 0.015 & $-0.001(0.001)$ & - & 0.21 \\
\hline Smoking habit & $0.666(0.155)$ & $1.95(1.44,2.64)$ & 0.001 & $0.674(0.174)$ & $1.96(1.40,2.76)$ & 0.001 & $0.589(0.330)$ & $1.80(0.95,3.46)$ & 0.074 \\
\hline Log vitamin D & $-0.573(0.167)$ & $0.56(0.41,0.78)$ & 0.001 & $-0.554(0.193)$ & $0.58(0.39,0.84)$ & 0.004 & $-0.692(0.044)$ & $0.50(0.25,0.98)$ & 0.044 \\
\hline ABI (normal) & $-0.318(0.209)$ & $0.73(0.48,1.09)$ & 0.128 & $-0.428(0.245)$ & $0.65(0.40,1.05)$ & 0.08 & & & \\
\hline ABI (calcified) & $0.460(0.338)$ & $1.58(0.82,3.11)$ & 0.174 & $0.675(0.427)$ & $1.96(0.87,4.64)$ & 0.114 & & & \\
\hline Diabetes & $0.572(0.169)$ & $1.77(1.28,2.48)$ & 0.001 & & & & & & \\
\hline Group CKD 4-5 & $0.114(0.150)$ & $1.12(0.84,1.51)$ & 0.446 & $0.228(0.172)$ & $1.26(0.90,1.76)$ & 0.184 & & & \\
\hline Group RRT & $0.930(0.216)$ & $2.54(1.67,3.90)$ & 0.001 & $0.982(0.235)$ & $2.67(1.70,4.27)$ & 0.001 & & & \\
\hline Age: gender & $0.029(0.013)$ & - & 0.024 & $0.047(0.014)$ & - & 0.001 & & & \\
\hline
\end{tabular}

na, not applicable. RRT, renal replacement therapy. ABI, ankle brachial index.

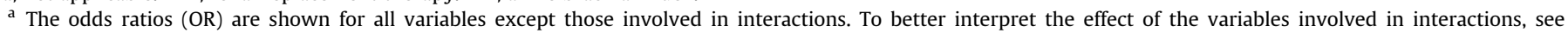
Supplementary figure 1 . 
DM patients $(59.9 \%$ vs. $42.7 \%, p=0.001)$ compared with non-diabetic patients. However, no differences in the frequency of femoral plaque were found between groups. Women with diabetes presented more frequently with a plaque at any vascular site $(69.7 \% \mathrm{vs}$. $53.8 \%, p=0.001)$ and plaques in more than two territories (52.1\% vs. $37.6 \%, p=0.003$ ) than women without diabetes. Interestingly, they presented a similar prevalence of plaque as men without diabetes (69.7\% vs. 71\%) (Table 2).

\subsection{Factors associated with the presence of plaques at baseline}

In the multivariable analysis of the whole population presence of plaques was significantly associated with age, smoking, receiving RRT and DM (Supplementary table 1). There was an interaction between age and gender, being a higher risk of plaque presence at older ages, increasing faster for men (Supplementary figure 1). Among non-diabetic patients, plaque was associated with age, gender, smoking and receiving RRT. Similar to the model for all population, the risk of plaque increased with aging, and was faster in men than in women. In patients with DM, age and gender were positively associated with presence of plaques, and an increased risk with aging, lessened at older ages, was also observed. Additionally, 25-OH vitamin D serum concentrations were inversely associated with the presence of plaque in both CKD patients with and without diabetes (OR 0.50, $p=0.044$; OR 0.58, $p=0.004$, respectively) (Table 3 ).

\subsection{Progression of atheromatous disease at 24 months}

Following a 24 month-period, carotid plaques were found in $63.1 \%$ of non-diabetic patients and in $83.8 \%$ of patients with DM $(p<0.001)$. Femoral plaques were described in $65 \%$ of non-diabetic patients versus $79 \%$ of patients with DM $(p<0.001)$. Along with these findings, presence of plaque in both vascular territories, carotid and femoral, was found in $49.8 \%$ of patients without DM compared to a $69 \%$ among patients with $\mathrm{DM}(p<0.001)$. Finally, the proportion of patients with femoral plaque(s) without carotid plaque was higher in diabetic patients compared with non-diabetic patients. However, no differences were observed in the proportion of patients with carotid plaque(s) without femoral plaque between patients with or without diabetes. This finding was confirmed for both women and men (Table 4). A greater proportion of plaque progression was found in the common femoral artery and carotid bulb territories (Supplementary table 2).

\subsection{Factors associated with progression of atheromatous disease at 24 months}

In the logistic regression models of the whole population, plaque progression at 24 months was strongly and positively associated with the number of territories with plaque(s) at baseline, age, smoking, RRT and DM (Supplementary table 3). We found a statistically significant interaction between the number of territories with plaque at baseline and age (Table 5). We observed that the progression of atherosclerosic disease over time depends on the number of territories affected at baseline. Thus, the higher the number of territories affected at the beginning of the study, the lower the probability of progression of the atherosclerotic plaque at follow-up (lower potential number of newly affected territories). On the contrary, having a low number of affected territories at baseline is associated with a higher risk of atherosclerosis progression (higher potential number of new territories affected that were initially free of plaques) (Supplementary figure 2). Among patients without DM, progression of atheromatous disease was associated with age, number of territories with plaque at baseline,

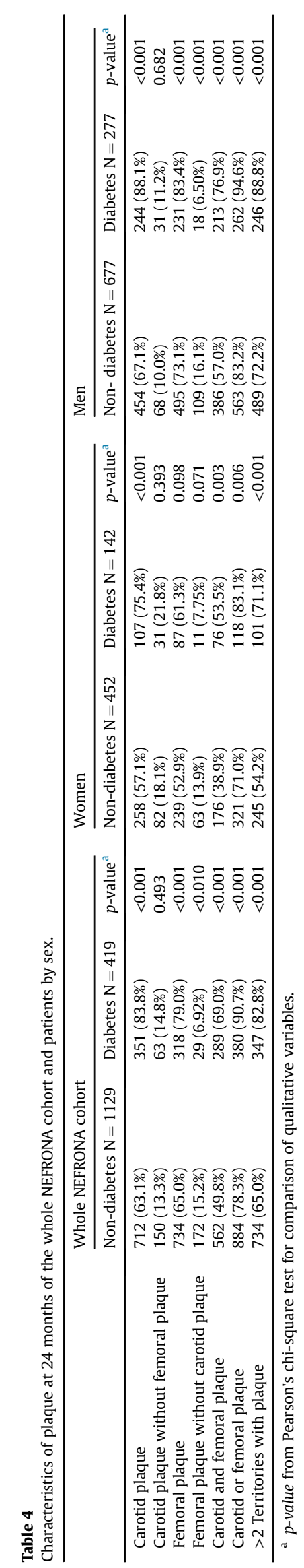


Table 5

Multivariate logistic regression model for the progression of plaque at 24 months in the whole NEFRONA cohort, CKD without diabetes and CKD with diabetes.

\begin{tabular}{|c|c|c|c|c|c|c|c|c|c|}
\hline & \multicolumn{3}{|c|}{ Whole NEFRONA cohort } & \multicolumn{3}{|c|}{ CKD without diabetes } & \multicolumn{3}{|c|}{ CKD with diabetes } \\
\hline & Estimate (SE) & OR $(95 \% \mathrm{CI})^{\mathrm{a}}$ & $p$-value & Estimate (SE) & OR $(95 \% \mathrm{CI})^{\mathrm{a}}$ & $p$-value & Estimate (SE) & OR $(95 \% \mathrm{CI})^{\mathrm{a}}$ & $p$-value \\
\hline Intercept & $-6.340(0.978)$ & na & 0.001 & $-6.604(1.116)$ & na & 0.001 & $-4.669(0.963)$ & na & 0.001 \\
\hline Age & $0.071(0.007)$ & -- & 0.001 & $0.066(0.008)$ & -- & 0.001 & $0.088(0.016)$ & -- & 0.001 \\
\hline Territories with basal plaque & $0.967(0.182)$ & -- & 0.001 & $0.932(0.210)$ & -- & 0.001 & $1.145(0.387)$ & -- & 0.003 \\
\hline Smoker & $0.514(0.119)$ & $1.67(1.33,2.11)$ & 0.001 & $0.496(0.135)$ & $1.64(1.26,2.14)$ & 0.001 & $0.583(0.250)$ & $1.79(1.10,2.94)$ & 0.02 \\
\hline Group CKD 4-5 & $0.115(0.125)$ & $1.12(0.88,1.43)$ & 0.358 & $0.146(0.144)$ & $1.16(0.87,1.54)$ & 0.314 & $0.041(0.249)$ & $1.04(0.64,1.70)$ & 0.87 \\
\hline Group RRT & $0.712(0.173)$ & $2.04(1.46,2.87)$ & 0.001 & $0.670(0.188)$ & $1.95(1.36,2.84)$ & 0.001 & $0.921(0.448)$ & $2.51(1.09,6.41)$ & 0.04 \\
\hline Diastolic blood pressure & $0.005(0.005)$ & $1.01(0.99,1.02)$ & 0.372 & $0.005(0.006)$ & $1.01(0.99,1.02)$ & 0.401 & & & \\
\hline Log pulse pressure & $0.425(0.220)$ & $1.53(0.99,2.36)$ & 0.054 & $0.547(0.256)$ & $1.73(1.05,2.86)$ & 0.032 & & & \\
\hline Diabetes & $0.587(0.139)$ & $1.80(1.37,2.37)$ & 0.001 & & & & & & \\
\hline Age: territories with basal plaque & $-0.016(0.003)$ & - & 0.001 & $-0.015(0.003)$ & -- & 0.001 & $-0.018(0.006)$ & -- & 0.002 \\
\hline
\end{tabular}

na, not applicable. CKD, chronic kidney disease; RRT, renal replacement therapy.

${ }^{a}$ The odds ratios (OR) are shown for all variables except those involved in interactions. To better interpret the effect of the variables involved in interactions, see Supplementary figure 2 .

smoking, pulse pressure and receiving RRT. In diabetic patients, disease progression was associated with the same variables as in non-diabetic patients except for pulse pressure. The interaction between the number of territories with plaque at baseline and age was also significant in these two models (Table 5).

\section{Discussion}

The present study evaluated the differences in the presence and progression of subclinical atherosclerosis between CKD patients with and without DM in a large cohort of subjects without previous CV events. We found that CKD patients with DM presented a higher frequency and burden of atherosclerotic plaques in different vascular territories compared to non-diabetic patients. The progression of atherosclerosis was also more rapid in subjects with DM. Additionally, we found that factors associated with the presence and progression of atheromatous disease differed between patients with and without DM, whereas factors associated with atheromatous disease progression were the same for CKD patients with or without DM with the exception of pulse pressure, that did not turn out to be a risk factor in patients with DM.

A higher frequency of carotid plaques has already been described in subjects with T2D at disease onset in Spain [16]. In CKD, there is also an increased prevalence of $A D$ [34,35], even at earlier stages of impaired kidney function. However, very few data exist regarding the specific prevalence of subclinical atheromatous disease in patients with both CKD and diabetes. Our group recently described a two-fold higher risk of subclinical atherosclerosis in patients with diabetic nephropathy compared with kidney disease from other causes [36]. In the present study, we observed a substantially higher prevalence of carotid and femoral plaques in patients with CKD and DM than in their counterparts without DM, was well as a higher burden of atheromatous disease (i.e., $>2$ territories with plaques).

Several studies have examined CVD at different vascular territories in general population cohorts $[10,19,23,37]$. Those studies performed in middle-aged cohorts have shown that femoral arteries are the vascular territory most frequently found to have plaques. They have also demonstrated that scanning just the carotid or just the femoral arteries predicted fewer events than examining both territories $[17,23,38]$. Conversely, in our study, plaques were substantially more common in the carotid arteries than in the femoral arteries in patients with DM. Whereas a similar proportion of patients with plaque at either carotid or femoral territory was observed in patients without diabetes. Although femoral plaques were more frequent in patients with DM, there were no differences in femoral plaque frequency between groups in the absence of carotid lesions. These data indicate that factors playing a role in femoral plaque formation might be different from those related to carotid plaque formation. Notably, these findings were consistent also when stratified by gender.

Male gender is a well-known cardiovascular risk factor. However, previous studies have shown that women with DM do not present the CV-protective gender effect $[39,40]$. In a recent crosssectional study, Catalan et al. [16] reported that women with DM presented more advanced carotid lesions than men with DM when compared with their respective counterparts without diabetes. In line with these results, in our study, women with DM presented a similar prevalence of atheromatous lesions as men without DM. Moreover, while in women the higher differential risk associated with diabetes remained constant independently of age, in men the risk associated with diabetes was attenuated with increasing age (Supplementary figure 1). All these findings support the more deleterious effect of diabetes in women.

In general population studies, the presence of atherosclerotic plaques either at carotid or femoral territories has been described to be associated with traditional cardiovascular risk factors $[2,4,17,41]$. In patients with DM, a study assessing atherosclerosis in a new-onset T2D cohort free from previous CVD reported that age, gender, HbA1c, triglycerides, HDL cholesterol and hypertension were associated with carotid plaques [16]. However, in CKD patients, baseline data from the NEFRONA study showed that at any given CKD stage, older age, male gender, DM and smoking were independently associated with the presence of plaque [35]. Our study found that in CKD patients without DM, the presence of plaque was significantly associated with age, male gender, smoking and dialysis, whereas among patients with both CKD and DM, only age and male gender were independent factors for the presence of plaques. This finding suggests that DM is a potent risk condition for atheromatous disease that outweighs predominant CV risk factors such as smoking and dialysis. On the other hand, our data also suggest that vitamin $\mathrm{D}$ may protect against atheromatous disease in CKD patients as it has already been reported in previous data from the NEFRONA cohort [30].

Concerning risk factors associated with the progression of atherosclerosis, age, smoking, total cholesterol and systolic blood pressure have been described to predict the progression of extracoronary atherosclerosis in elderly patients [42], middle-age subjects [3] and the general population [43]. Very few studies have evaluated the progression of atherosclerosis in CKD patients. Presence and number of plaques at baseline is among the factors described to be associated with progression of atherosclerosis, both in the NEFRONA cohort as well as in patients with end-stage renal disease $[19,30]$. Plaque at baseline was one of the factors that 
predicted the progression of atherosclerosis in CKD patients of the NEFRONA cohort [30]. In relation to DM and CKD, lower GFR was reported to be associated with the progression of carotid plaques [44]. Data regarding AD progression in the present study showed that factors associated with plaque progression are very similar in patients with CKD with or without DM (age, plaque at baseline, smoking, RRT), with the exception of pulse pressure, which is associated with progression only in those without DM. Smoking and RRT are associated with atheromatous disease progression but not with the presence of atherosclerotic plaque in patients with CKD and DM.

Our study limitations included the absence of precise phenotyping of the type of DM. Initially, the NEFRONA study was designed to investigate renal disease and CV risk. Therefore, data on clinical characteristics relevant to DM at baseline are not complete. Another limitation is the substantial number of dropouts due to death, CVD or renal allograft. Additionally, the length of the followup was limited to 24 months, and a more prolonged period of follow-up could have provided more data on plaque progression. However, the current results will be hopefully complemented with information on clinical events in future studies.

Overall, our results show that subclinical atherosclerosis is more frequent and rapidly progressive in patients with CKD and DM than in CKD patients without DM. Moreover, diabetes is such a strong risk factor for $A D$ that it outweighs other described risk factors associated with the presence of atherosclerosis in CKD patients without DM. Therefore, this suggests a synergistic detrimental effect of these two conditions; individuals with both CKD and DM are at an extraordinarily increased risk of atherosclerotic disease. For this reason, a more targeted and aggressive therapeutic approach beginning at the very earliest stages in patients with CKD and DM appears to be reasonable in order to prevent atheromatous disease.

\section{Conflicts of interest}

The authors declared they do not have anything to disclose regarding conflict of interest with respect to this manuscript.

\section{Financial support}

This research was supported by grants from the Carlos III National Institute of Health (PI14/1772), the European Foundation for the study of diabetes (2014-EFSD-00914) and European Regional Development Fund. CIBER for Diabetes and Associated Metabolic Diseases (CIBERDEM) is an initiative of ISCIII, Spain. The NEFRONA study is funded by a research grant from AbbVie.

\section{Author contributions}

A.P. and E. C. contributed to the study design, conduct of the study, data analysis, and writing of the manuscript. JM.V., M.B. and A.B. contributed to data collection and conduct of the study. H.P. and X.D. contributed to data analyses and writing of the manuscript. E.F., P-H. G. and M.P-D. contributed to data interpretation and discussion N.A. and D.M. contributed to the study design and coordination, conduct of the study, data analysis, and writing of the manuscript. All authors critically reviewed the manuscript and approved the final version for publication. D.M. and N.A. are the guarantors of this work and, as such, had full access to all the data in the study and takes responsibility for the integrity of the data and the accuracy of the data analysis.

\section{Acknowledgments}

The authors would like to thank the NEFRONA team (Teresa
Vidal, Eva Castro, Virtudes María, Teresa Molí, Meritxell Soria), and the Biobank of RedInRen for their invaluable support.

\section{Appendix A. Supplementary data}

Supplementary data related to this article can be found at https://doi.org/10.1016/j.atherosclerosis.2018.07.018.

\section{References}

[1] A.S. Go, G.M. Chertow, D. Fan, C.E. McCulloch, C.Y. Hsu, Chronic kidney disease and the risks of death, cardiovascular events, and hospitalization, N. Engl. J. Med. 351 (2004) 1296-1305.

[2] M. Afkarian, M.C. Sachs, B. Kestenbaum, I.B. Hirsch, K.R. Tuttle, J. Himmelfarb, I.H. de Boer, Kidney disease and increased mortality risk in type 2 diabetes, J. Am. Soc. Nephrol. 24 (2013) 302-308.

[3] M.F. Piepoli, A.W. Hoes, S. Agewall, European Guidelines on cardiovascular disease prevention in clinical practice, Eur. Heart J. 37 (2016) 2291-2293.

[4] K. Kalantar-Zadeh, G. Block, M.H. Humphreys, J.D. Kopple, Reverse epidemiology of cardiovascular risk factors in maintenance dialysis patients, Kidney Int. 3 (2003) 793-808.

[5] T. Tillin, A.D. Hughes, P. Whincup, J. Mayet, N. Sattar, P.M. McKeigue N. Chaturvedi, SABRE Study Group, Ethnicity and prediction of cardiovascular disease: performance of QRISK2 and Framingham scores in a U.K. tri-ethnic prospective cohort study, Heart 100 (2014) 60-67.

[6] M.A. Salinero-Fort, F.J. San Andrés-Rebollo, C. de Burgos-Lunar, J.C. AbánadesHerranz, E. Carrillo-de-Santa-Pau, R.M. Chico-Moraleja, R. Jiménez-García, A. López-de-Andrés, P. Gómez-Campelo, MADIABETES Group, Cardiovascular and all-cause mortality in patients with type 2 diabetes mellitus in the MADIABETES Cohort Study: association with chronic kidney disease, J. Diabet. Complicat. 30 (2016) 227-236.

[7] T. Ninomiya, V. Perkovic, B.E. de Galan, S. Zoungas, A. Pillai, M. Jardine, A. Patel A. Cass, B. Neal, N. Poulter, C.E. Mogensen, M. Cooper, M. Marre, B. Williams, P. Hamet, G. Mancia, M. Woodward, S. Macmahon, J. Chalmers, ADVANCE Collaborative Group, Albuminuria and kidney function independently predict cardiovascular and renal outcomes in diabetes, J. Am. Soc. Nephrol. 20 (2009) $1813-1821$.

[8] N. Alonso, A. Traveset, E. Rubinat, E. Ortega, N. Alcubierre, J. Sanahuja, M. Hernández, A. Betriu, C. Jurjo, E. Fernández, D. Mauricio, Type 2 diabetesassociated carotid plaque burden is increased in patients with retinopathy compared to those without retinopathy, Cardiovasc. Diabetol. 14 (2015) 33.

[9] G. Targher, G. Zoppini, W. Mantovani, M. Chonchol, C. Negri, V. Stoico, A. Mantovani, F. De Santi, E. Bonora, Comparison of two creatinine-based estimating equations in predicting all-cause and cardiovascular mortality in patients with Type 2 diabetes, Diabetes Care 35 (2012) 2347-2353.

[10] K. Matsushita, J. Coresh, Y. Sang, J. Chalmers, C. Fox, E. Guallar, T. Jafar, S.K. Jassal, G.W. Landman, P. Muntner, P. Roderick, Estimated glomerular filtration rate and albuminuria for prediction of cardiovascular outcomes: a collaborative meta-analysis of individual participant data, Lancet Diabetes Endocrinol 3 (2014) 514-525.

[11] T. Nakano, T. Ninomiya, S. Sumiyoshi, H. Fuji, Y. Doi, H. Hirakata, K. Tsuruya, M. Iida, Y. Kiyohara, K. Sueishi, Association of kidney function with coronary atherosclerosis and calcification in autopsy samples from Japanese elders: the Hisayama Study, Am. J. Kidney Dis. 55 (2010) 21-30.

[12] P.A. McCullough, S. Li, C.T. Jurkovitz, L. Stevens, A.J. Collins, S.C. Chen, K.C. Norris, S. McFarlane, B. Johnson, M.G. Shlipak, C.I. Obialo, W.W. Brown, J. Vassalotti, A.T. Whaley-Connell, R.M. Brenner, G.L. Bakris, KEEP Investigators, Chronic kidney disease, prevalence of premature cardiovascular disease, and relationship to short-term mortality, Am. Heart J. 156 (2008) $277-283$.

[13] P. Muntner, J. He, L. Hamm, C. Loria, P.K. Whelton, Renal insufficiency and subsequent death resulting from cardiovascular disease in the United States, J. Am. Soc. Nephrol. 13 (2002) 745-753.

[14] M.C. Thomas, M.E. Cooper, P. Zimmet, Changing epidemiology of type 2 diabetes mellitus and associated chronic kidney disease, Nat. Rev. Nephrol. 12 (2016) 73-81.

[15] P.H. Groop, M.C. Thomas, J.L. Moran, J. Wadèn, L.M. Thorn, V.P. Mäkinen M. Rosengård-Bärlund, M. Saraheimo, K. Hietala, O. Heikkilä, C. Forsblom, FinnDiane Study Group, The presence and severity of chronic kidney disease predicts all-cause mortality in type 1 diabetes, Diabetes 58 (2009) 1651-1658.

[16] M. Catalan, Z. Herreras, M. Pinyol, A. Sala-Vila, A.J. Amor, E. de Groot, R. Gilabert, E. Ros, E. Ortega, Prevalence by sex of preclinical carotid atherosclerosis in newly diagnosed type 2 diabetes, Nutr. Metabol. Cardiovasc. Dis. 25 (2015) 742-748

[17] L. Fernández-Friera, J.L. Peñalvo, A. Fernández-Ortiz, B. Ibanez, B. LopezMelgar, M. Laclaustra, B. Oliva, A. Mocoroa, J. Mendiguren, V. Martinez de Vega, L. Garcia, J. Molina, J. Sanchez-Gonzalez, G. Guzman, J.C. Alonso-Farto E. Guallar, F. Civeira, H. Sillesen, S. Pocock, J.M. Ordovas, G. Sanz, L.J. JimenezBorreguero, V. Fuster, Prevalence, vascular distribution, and multiterritorial extent of subclinical atherosclerosis in a middle-aged cohort: the PESA (progression of early subclinical atherosclerosis) study, Circulation 131 (2015) 
$2104-2113$.

[18] Y. Inaba, J.A. Chen, S.R. Bergmann, Carotid plaque, compared with carotid intima-media thickness, more accurately predicts coronary artery disease events: a meta-analysis, Atherosclerosis 220 (2012) 128-133.

[19] F.A. Benedetto, G. Tripepi, F. Mallamaci, C. Zoccali, Rate of atherosclerotic plaque formation predicts cardiovascular events in ESRD, J. Am. Soc. Nephrol. 19 (2008) 757-763.

[20] T. Wannarong, G. Parraga, D. Buchanan, A. Fenster, A.A. House, D.G. Hackam, J.D. Spence, Progression of carotid plaque volume predicts cardiovascular events, Stroke 44 (2013) 1859-1865.

[21] U. Baber, R. Mehran, S. Sartori, M.M. Schoos, H. Sillesen, P. Muntendam, M.J. Garcia, J. Gregson, S. Pocock, E. Falk, V. Fuster, Prevalence, impact, and predictive value of detecting subclinical coronary and carotid atherosclerosis in asymptomatic adults: the BioImage study, J. Am. Coll. Cardiol. 65 (2015) 1065-1074.

[22] M.G. Silverman, J.R. Harkness, R. Blankstein, M.J. Budoff, A.S. Agatston, J.J. Carr, J.A. Lima, R.S. Blumenthal, K. Nasir, M.J. Blaha, Baseline subclinical atherosclerosis burden and distribution are associated with frequency and mode of future coronary revascularization: multi-ethnic study of atherosclerosis. JACC, Cardiovasc. Imag. 7 (2014) 476-486.

[23] A.E. Zavodni, B.A. Wasserman, R.L. McClelland, A.S. Gomes, A.R. Folsom J.F. Polak, J.A. Lima, D.A. Bluemke, Carotid artery plaque morphology and composition in relation to incident cardiovascular events: the Multi-Ethnic Study of Atherosclerosis (MESA), Radiology 271 (2014) 381-389.

[24] T. Rundek, H. Arif, B. Boden-Albala, M.S. Elkind, M.C. Paik, R.L. Sacco, Carotid plaque, a subclinical precursor of vascular events: the Northern Manhattan Study, Neurology 70 (2008) 1200-1207.

[25] M. Laclaustra, J.A. Casasnovas, A. Fernández-Ortiz, V. Fuster, M. León-Latre, L.J. Jiménez-Borreguero, M. Pocovi, Y. Hurtado-Roca, J.M. Ordovas, E. Jarauta, E. Guallar, B. Ibañez, F. Civeira, Femoral and carotid subclinical atherosclerosis association with risk factors and coronary calcium: the AWHS Study, J. Am. Coll. Cardiol. 67 (2016) 1263-1274.

[26] M. Junyent, M. Martínez, M. Borràs, B. Coll, J.M. Valdivielso, T. Vidal, F. Sarró, J. Roig, L. Craver, E. Fernández, Predicting cardiovascular disease morbidity and mortality in chronic kidney disease in Spain. The rationale and design of NEFRONA: a prospective, multicenter, observational cohort study, BMC Nephrol. 11 (2010) 14.

[27] M. Junyent, M. Martínez, M. Borrás, A. Bertriu, B. Coll, L. Craver, M.P. Marco, F. Sarró, J.M. Valdivielso, E. Fernández, Usefulness of imaging techniques and novel biomarkers in the prediction of cardiovascular risk in patients with chronic kidney disease in Spain: the NEFRONA project, Nefrologia 30 (2010) $119-126$.

[28] D. Arroyo, A. Betriu, M. Martinez-Alonso, T. Vidal, J.M. Valdivielso, E. Fernández, investigators from the NEFRONA study, Observational multicenter study to evaluate the prevalence and prognosis of subclinical atheromatosis in a Spanish chronic kidney disease cohort: baseline data from the NEFRONA study, BMC Nephrol. 15 (2014) 168.

[29] B. Coll, A. Betriu, M. Martínez-Alonso, M.L Amoedo, MV. Arcidiacono, M. Borras, J.M. Valdivielso, E. Fernández, Large artery calcification on dialysis patients is located in the intima and related to atherosclerosis, Clin. J. Am. Soc. Nephrol. 6 (2011) 303-310.

[30] M. Gracia, À. Betriu, M. Martínez-Alonso, D. Arroyo, M. Abajo, E. Fernández, J.M. Valdivielso, NEFRONA Investigators, Predictors of subclinical atheromatosis progression over 2 years in patients with different stages of CKD, Clin. J. Am. Soc. Nephrol. 11 (2016) 287-296.

[31] J.H. Stein, C.E. Korcarz, R.T. Hurst, E. Lonn, C.B. Kendall, E.R. Mohler, S.S. Najjar C.M. Rembold, W.S. Post, American society of echocardiography carotid intima-media thickness task force: a consensus statement from the American society of echocardiography carotid intima-media thickness task force, J. Am. Soc. Echocardiogr. 21 (2008) 93-111.

[32] P.J. Touboul, M.G. Hennerici, S. Meairs, H. Adams, P. Amarenco, M. Desvarieux, S. Ebrahim, M. Fatar, R. Hernandez, S. Kownator, P. Prati, T. Rundek, A. Taylor, N. Bornstein, L. Csiba, E. Vicaut, K.S. Woo, F. Zannad, Mannheim intima-media thickness consensus, Cerebrovasc. Dis. 18 (2004) 346-349.

[33] M.C. Tattersall, A. Gassett, C.E. Korcarz, A.D. Gepner, J.D. Kaufman, K.J. Liu, B.C. Astor, L. Sheppard, R.A. Kronmal, J.H. Stein, Predictors of carotid thickness and plaque progression during a decade: the Multi-Ethnic Study of Atherosclerosis, Stroke 45 (2014) 3257-3262.

[34] G.A. Adeseun, D. Xie, X. Wang, M.M. Joffe, E.R. Mohler, R.R. Townsend, M. Budoff, S.E. Rosas, Carotid plaque, carotid intima-media thickness, and coronary calcification equally discriminate prevalent cardiovascular disease in kidney disease, Am. J. Nephrol. 36 (2012) 342-347.

[35] A. Betriu, M. Martinez-Alonso, M.V. Arcidiacono, J. Cannata-Andia, J. Pascual, J.M. Valdivielso, E. Fernández, Investigators from the NEFRONA Study, Prevalence of subclinical atheromatosis and associated risk factors in chronic kidney disease: the NEFRONA study, Nephrol. Dial. Transplant. 29 (2014) 1415-1422.

[36] C. Barrios, J. Pascual, S. Otero, M.J. Soler, E. Rodríguez, S. Collado, A. Faura, S. Mojal, J.F. Navarro-González, A. Betriu, E. Fernandez, J.M. Valdivielso, Investigators of the NEFRONA study, Diabetic nephropathy is an independent factor associated to severe subclinical atheromatous disease, Atherosclerosis 242 (2015) 37-44.

[37] C. Lamina, C. Meisinger, I.M. Heid, H. Löwel, B. Rantner, W. Koenig, F. Kronenberg, Kora Study Group, Association of ankle-brachial index and plaques in the carotid and femoral arteries with cardiovascular events and total mortality in a population-based study with 13 years of follow-up, Eur. Heart J. 27 (2006) 2580-2587.

[38] G. Belcaro, A.N. Nicolaides, G. Ramaswami, M.R. Cesarone, M. De Sanctis, L. Incandela, P. Ferrari, G. Geroulakos, A. Barsotti, M. Griffin, S. Dhanjil, M. Sabetai, M. Bucci, G. Martines, Carotid and femoral ultrasound morphology screening and cardiovascular events in low risk subjects: a 10-year follow-up study (the CAFES-CAVE study), Atherosclerosis 156 (2001) 379-387.

[39] R. Huxley, F. Barzi, M. Woodward, Excess risk of fatal coronary heart disease associated with diabetes in men and women: meta-analysis of 37 prospective cohort studies, BMJ 332 (2006) 73-78.

[40] Emerging Risk Factors Collaboration, N. Sarwar, P. Gao, S.R. Seshasai, R. Gobin, S. Kaptoge, E. Di Angelantonio, E. Ingelsson, D.A. Lawlor, E. Selvin, M. Stampfer, C.D. Stehouwer, S. Lewington, L. Pennells, A. Thompson, N. Sattar, I.R. White, K.K. Ray, J. Danesh, Diabetes mellitus, fasting blood glucose concentration, and risk of vascular disease: a collaborative metaanalysis of 102 prospective studies, Lancet 375 (2010) 2215-2222.

[41] R. Sturlaugsdottir, T. Aspelund, G. Bjornsdottir, S. Sigurdsson, B. Thorsson, G. Eiriksdottir, V. Gudnason, Prevalence and determinants of carotid plaque in the cross-sectional REFINE-Reykjavik study, BMJ. Open 6 (2016), e012457.

[42] I.M. van der Meer, A. Iglesias del Sol, A.E. Hak, M.L. Bots, A. Hofman, J.C. Witteman, Risk factors for progression of atherosclerosis measured at multiple sites in the arterial tree: the Rotterdam Study, Stroke 34 (2003) 2374-2379.

[43] E.B. Mathiesen, S.H. Johnsen, T. Wilsgaard, K.H. Bønaa, M.L. Løchen, I. Njølstad, Carotid plaque area and intima-media thickness in prediction of first-ever ischemic stroke: a 10-year follow-up of 6584 men and women: the Tromsø Study, Stroke 42 (2011) 972-978.

[44] L.E. Wagenknecht, J. Divers, T.C. Register, G.B. Russell, D.W. Bowden, J. Xu, C.D. Langefeld, L. Lenchik, K.A. Hruska, J.J. Carr, B.I. Freedman, Bone mineral density and progression of subclinical atherosclerosis in African-Americans with Type 2 diabetes, J. Clin. Endocrinol. Metab. 101 (2016) 4135-4141. 